\title{
QoS Performance Analysis of Cognitive Radio-based Virtual Wireless Networks
}

\author{
Brent Ishibashi \\ Cheriton School of Computer Science \\ University of Waterloo \\ Waterloo, Canada \\ Email: bkishiba@uwaterloo.ca
}

\author{
Nizar Bouabdallah \\ INRIA-IRISA \\ Campus universitaire de Beaulieu \\ 35042 Rennes, Cedex, France \\ Email: nizar.bouabdallah@inria.fr
}

\author{
Raouf Boutaba \\ Cheriton School of Computer Science \\ University of Waterloo \\ Waterloo, Canada \\ Email: rboutaba@uwaterloo.ca
}

\begin{abstract}
Cognitive radio presents a new approach to wireless spectrum utilization and management. In this work, the potential performance improvement gained by applying cognitive radio to multiple-provider wireless systems is investigated. It is shown that virtual wireless networks can be created, utilizing only the residual wasted bandwidth of the primary service providers. These virtual networks are able to support large volumes of users, while still ensuring that QoS reliability requirements, such as blocking and dropping guarantees, are achieved. A Markov chain-based analysis of classic and cognitive systems is complemented by simulations in order to present a quantified perspective of the potential benefits of cognitive radio techniques.
\end{abstract}

Index Terms - Cognitive Networks, wireless networks, virtual networks, performance analysis, service reliability

\section{INTRODUCTION}

Despite the perceived scarcity of wireless spectrum, a scenario has emerged where, due to the fixed allocation of frequency bands, overall spectrum utilization is relatively low [1]. Although certain frequencies experience severe congestion, in many locations large holes of unused spectrum exist. Therefore, regulators have realized that there is a need for an improved, more dynamic and efficient, mechanism for management of wireless resources [2]. This has lead to a tremendous interest in Cognitive Radio (CR), whose frequency agility gives it the ability to flexibly change channels and technologies. This allows a device to adaptively utilize whatever wireless resources are available at its time and place, taking advantage of spectrum holes that would otherwise be wasted.

In this work, we consider the potential improvements that could be gained by utilizing cognitive radios in the presence of conventional call-based primary users. However, rather than only considering opportunistic best-effort traffic, we explore the ability of cognitive radio to provide sufficient capacity to support additional QoS traffic. Using classical service reliability metrics (call dropping, call blocking, and resource utilization), analytical and simulation models have been developed to in order to evaluate the system performance.

Using these models, we consider the creation of virtual wireless networks (VWN). It is shown that using CR techniques, wireless network operators can loan or lease their resources to virtual operators, creating additional networks to serve additional clients. Moreover, it is also shown that these improvements can be achieved without impacting the original primary clients, and that these new networks can still make and fulfil reasonable QoS guarantees. Therefore, virtual operators can create a feasible QoS network by leasing residual, unused bandwidth from real service providers.

\section{Cognitive Radio And Dynamic Spectrum ALLOCATION}

Cognitive Radio represents a long-term goal for making wireless communication more intelligent and efficient. The term was initially applied to extending software radios with a selfawareness about its characteristics and requirements, in order to determine an appropriate radio etiquette to be used [3]. This self-awareness includes an awareness of its environment, through the monitoring, modelling, and prediction of the radio spectrum [4]. However, while cognitive radio encapsulates many different concepts into a single vision, early work has latched onto one aspect of $\mathrm{CR}$ in particular - the idea of dynamic management of the wireless spectrum [5] [6].

Opening regulated spectrums has raised concerns from primary users and providers. As a resource, spectrum is extremely valuable, and many licensees have invested heavily in acquiring their spectrum rights [7] [8]. Many are therefore very hesitant to allow sharing of their frequency bands with secondary "cognitive radio" users. However, due to the need to maintain QoS, even heavily loaded networks often have residual capacity available. CR could offer considerable value to service providers, by allowing them to increase spectrum utilization and efficiency, improve their ability to service their own clients, or lease their residual (unused) bandwidth to other parties.

Previous works addressing the capacity of cognitive radio systems have focused on the information theory perspective at the physical level [9] [10]. In this work, a higher level call model is considered, at a single location. The system is evaluated in terms of classical QoS reliability metrics, as well as resource utilization. The approach used has similarities to those seen in works on spectrum pooling [11]. In [12], an analysis is performed on a spectrum pooling system, however it is limited to a single license owner, supporting additional users (renters). [13] also applies continuous time Markov chains to dynamic spectrum access, however, it focuses on the interactions between only a single primary user and additional unlicensed users on a single network.

This work differs in several ways. First, multiple networks (license owners) are considered. This allows for the direct comparison of cognitive with non-cognitive systems, without any additional traffic, showing the clear benefit of the cognitive abilities. Second, this work explores the interactions of cognitive and non-cognitive users within the networks, and shows the differentiation in the QoS delivered to each type of user.

Finally, the concepts of virtual wireless networks and virtual 


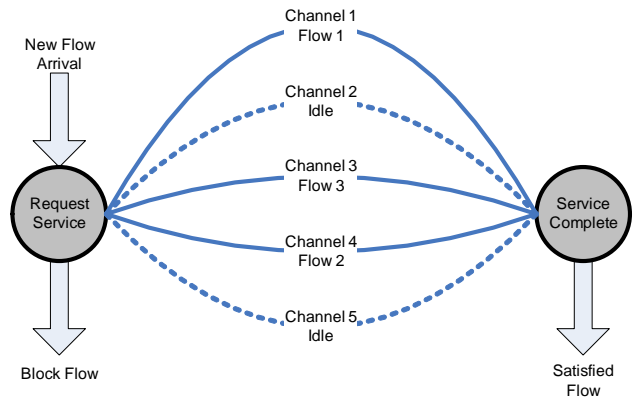

Fig. 1. A Network with 5 Channels

service providers are introduced. The term "virtual unlicensed spectrum" was used in [14]. However, that work describes the use of cognitive radio to re-use allocated (but unused) spectrum as if it were unlicensed. This work shows that service providers' unused bandwidth is still a valuable resource, and that a viable virtual operator (or in fact many) could be created, despite not having any dedicated wireless resources of its own.

\section{SYSTEM DESCRIPTION}

The system under consideration is a collection of one or more wireless access networks. In order to quantify the performance improvement gained by enabling cognitive radio capabilities, a classical network is first described, then extended to include the additional requirements and capabilities of cognitive radios.

\section{A. The Classic Network}

A network belonging to service provider $i$ possesses wireless resources in the form of $K_{i}$ channels. Connection-based traffic is considered, where service requirements of each flow or connection $c_{i}$ is continuous and constant. Flows arrive at the network and request service. Each flow requires the capacity of one channel for the duration of its service in order to fulfil its requirements. If the network has available resources, it can accept the flow. If not, then the flow must be blocked - service is refused and the flow ends without being serviced. Once accepted, a flow must have continuous service for the duration of its service time. The single network is depicted in Fig. 1.

The classical system consists of $N$ classical networks. Each network has its own set of channels, with the total capacity of the system being $M=\sum_{i=1}^{N} K_{i}$. However, these networks are completely independent of each other, with clients arriving to network $i$ only being serviced on network $i$. As a result, when a network reaches its capacity, it must start blocking flows, as it has no available channels. Therefore, flows may be blocked even if the overall utilization of the total system (the complete set of $N$ networks) is relatively low.

\section{B. The Cognitive Scenario}

This is where applying the Cognitive Radio concept can greatly benefit the system. In this work, CR traffic is studied with identical traffic users, being serviced by the existing providers. These resources could be used in other ways (e.g. peer-to-peer local communication), however, this approach allows a direct comparison to be made with the classic system.

Classical and cognitive traffic for network $i$ are distinguished as $c_{C L}(i)$ and $c_{C R}(i)$ respectively. For cognitive traffic, if resources are not available on its home network, the flow can switch to another network (operate on network $j, j \neq i$ ) if that network has an available channel. The traffic of each network in the system can be described in terms of two counts. Let $n_{C L}(i)$ and $n_{C R}(i)$ represent the number flows - classic and cognitive respectively - currently in service in the system belonging to network $i . n(i)$ is the sum of these counts, i.e., the total number of flows in the system belonging to network $i$.

Classic flows are still blocked if the home network is fully occupied by its own flows. Cognitive flows however are only blocked if the home network is fully occupied by its own flows and all other networks are also fully occupied. However, this creates a new possibility that may occur: the possibility of a dropped flow. Consider if a flow $c_{i}$ arrives to network $i$ while $\sum_{i=1}^{N}\left(n_{C L}(i)+n_{C R}(i)\right)=M$ (all channels on all networks are in use). If network $i$ is already using all of its own resources $\left(n_{C L}(i)+n_{C R}(i) \geq K_{i}\right)$, the flow must be blocked. However, if it has previously loaned its resources to be used for other networks' foreign traffic, it can now reclaim those resources for its own use, by forcing a foreign cognitive user $c_{C R}(j)$ $(j \neq i)$ off the channel.

As cognitive users may be pushed out of other networks and forced to return to their home network, an occupancy threshold $T h_{i}$ for classical users has also been included in the model. If the total number of network $i$ flows is greater than or equal to the threshold $\left(n(i) \geq T h_{i}\right)$ then arriving classic users are blocked.

\section{Virtual Wireless Networks}

Using the concept of a cognitive user, virtual wireless networks (VWNs) are a special case of this wireless network model. With no physical resources of its own, the VWN is a network $i$ with $K_{i}=0$. This means that a VWN does not service traffic on its own, instead relying on other (real) networks to provide service to its client flows.

Therefore, although a VWN has its own traffic, all of its flows must obviously be of cognitive type. Any classic flows would not be serviced, as there are never resources available for them. Cognitive flows however can be handled in the same way as they are in the cognitive networks. Any virtual network traffic forced off of another network must be dropped, as it can never pre-empt other traffic.

\section{AnAlytic And Simulation MOdELS}

\section{A. Analytic Model}

In order to analyze the behaviour of the system, the network is represented as $X(t)$, defined by the tuple $\left.\left(\left(n_{C L}(i), n_{C R}(i)\right), i=1, \ldots, N\right)\right)$ at time $t$. Flow arrivals are characterized by a set of Poisson processes with parameters $\lambda_{C L}(i)$ and $\lambda_{C R}(i)$ controlling the arrival rates for classic and cognitive flows. Similarly, $\mu_{C L}(i)$ and $\mu_{C R}(i)$ represent the exponentially distributed service rates. Therefore, $\{X(t), t \geq 0\}$ is a Markov process with continuous time and finite state space $\mathcal{S}=\left\{\left(n_{C L}(i), n_{C R}(i)\right), i=1, \ldots, N \mid n_{C L}(i) \leq\right.$ $\left.T h_{i}, \sum_{i=1}^{N} n(i) \leq M\right\}$.

The transition probability matrix $Q$ is derived for the process $X$ when leaving a generic state $s \in \mathcal{S}$, with $s=$ $\left(\left(n_{C L}(i), n_{C R}(i)\right), i=1, \ldots, N\right)$. Let $e$ denote the next 
occurring event and $s^{\prime} \in \mathcal{S}$ the associated new network state. $e$ can be either a new flow arrival or a flow departure. The transitions are found for both classic and cognitive flows. With all of the transition possibilities considered, the transition probability matrix $Q=\left[q_{s s^{\prime}}\right]$ can be derived. The steady state probabilities $\pi=\left[\pi_{s}\right]$ of the process $X$ is then obtained by resolving the following system:

$$
\pi Q=\pi \text { and } \sum_{s \in \mathcal{S}} \pi_{s}=1
$$

We have used three classical QoS metrics to evaluate the performance of the system: blocking probability, dropping probability, and failure probability. Each is calculated based on the state and transition probabilities as follows:

1) Blocking Probability: $P_{B}(i, C L)$ is the blocking probability for arriving classic flows belonging to network $i$. A classic flow $f$ is blocked if it arrives while its home network is already using more resources than its service threshold (i.e., $\left.n(i) \geq T h_{i}\right)$. Therefore, the probability of blocking is:

$$
P_{B}(i, C L)=\sum_{s \in \mathcal{S}} 1_{\mid n(i) \geq T h_{i}} \times \pi_{s}
$$

Similarly, $P_{B}(i, C R)$ is the blocking probability for cognitive flows. However, the conditions for blocking are different for these flows - in order to be blocked, two conditions must be true when the flow arrives: $i$ ) all network resources must be occupied $\left(\sum_{j=1}^{N} n(j)=M\right)$; and, ii) network $i$ already uses all of its own resources $\left(n(i) \geq K_{i}\right)$. Therefore, the blocking probability is:

$$
P_{B}(i, C R)=\left.\sum_{s \in \mathcal{S}} 1\right|_{\sum_{j=1}^{N} n(j)=M} \times 1_{\left.\right|_{n(i) \geq K_{i}}} \times \pi_{s}
$$

2) Dropping Probability: In-service classic connection are never dropped. Therefore, only $P_{D}(i, C R)$, the dropping probability of network $i$ 's cognitive flows, must be considered. Several conditions must occur in order for a cognitive flow to be dropped upon the arrival of a new flow $f$ belonging to network $j$. These conditions differ slightly, depending on whether the incoming flow is a classic $\left(c_{C L}(j)\right)$ or cognitive $\left(c_{C R}(j)\right)$ connection. In both cases, these two conditions must occur:

- All the $M$ channels in the system are occupied $\left(\sum_{k=1}^{N} n(k)=M\right)$. (i)

- Network $i$ is using more system resources than it physically possesses $\left(n(i)>K_{i}\right)$. (ii)

If $f$ is an arriving classic flow $\left(c_{C L}(j)\right)$, the following condition (iii) must also be true:

- Network $j$ uses less resources than its specified threshold $\left(n(j)<T h_{j}\right) .(i i i)$

Whereas if $f$ is an arriving cognitive flow $\left(c_{C R}(j)\right)$, condition $(i v)$ must occur:

- Network $j$ uses less resources than its physical capacity $\left(n(j)<K_{j}\right) .(i v)$

After calculating the probability of a flow being interrupted, the dropping probabilities for network $i$ are:

$$
P_{D}(i, C L)=0
$$

$$
\begin{aligned}
P_{D}(i, C R)= & {\left[\sum_{\substack{1 \leq j \leq N \\
j \neq i}} \sum_{s \in \mathcal{S}} 1_{\sum_{k=1}^{N} n(k)=M} \times\left.\right|_{\left.\right|_{n(i)>K_{i}}}\right.} \\
& \times 1_{\left.\right|_{n(j)<T h_{j}}} \times 1_{\left.\right|_{\lambda_{C L}(j)>0}} \times \pi_{s} \\
& \times \frac{Q\left(s, g_{C L}(s, i, j)\right)}{\lambda_{C R}(i)} \\
& +\sum_{\substack{1 \leq j \leq N \\
j \neq i}} \sum_{s \in \mathcal{S}} 1_{\sum_{k=1}^{N} n(k)=M} \times 1_{\mid n(i)>K_{i}} \\
& \times 1_{\mid n(j)<K_{j}} \times 1_{\lambda_{C R}(j)>0} \times \pi_{s} \\
& \left.\times \frac{Q\left(s, g_{C R}(s, i, j)\right)}{\lambda_{C R}(i)}\right] \\
& \times \frac{1}{1-P_{B}(i, C R)}
\end{aligned}
$$

3) Failure Probability: Cognitive traffic experiences a reduction in blocking, but an increase in dropping compared to classic traffic (where $P_{D}(i, C L)=0$ ). Therefore, $P_{F}$ is used to compare the overall performance of cognitive and classic traffic, where $P_{F}$ is the probability that an arriving flow will not receive the service it requires. $P_{F}$ is calculated using $P_{B}$ and $P_{D}$.

$$
P_{F}(i)=P_{B}(i)+\left(1-P_{B}(i)\right) \times P_{D}(i)
$$

For classic traffic, $P_{D}(i, C R)=0$, therefore $P_{F}(i, C L)$ is:

$$
P_{F}(i, C L)=P_{B}(i, C L)
$$

For cognitive traffic:

$$
P_{F}(i, C R)=P_{B}(i, C R)+\left(1-P_{B}(i, C R)\right) \times P_{D}(i, C R)
$$

\section{B. Simulation Model}

A simulation model has been developed in order to validate the analytic results. The system of networks was implemented as a discrete event simulation. Numerous evaluations were performed in order to confirm that the analytic results obtained in equations 2,3 , and 5 . In all cases, the results matched very closely.

For the simulations, the result obtained is dependent on the length of the simulation (the number of arrivals). Due to the presence of an initial transient effect caused by all networks starting in the 0-load state, simulations run for a short period of time have considerable error relative to the analytical result. However, as the number of arrivals increases, the result improves, reducing the error to an arbitrarily small value.

For the results presented in the next section, it has been confirmed that simulations have been run for a sufficient length of time, and that there is a good fit between the simulation and analytical results. Therefore, for presentation purposes, all figures show only the analytical results. Additionally, this section discusses only systems where all networks have the same number of channels (with the exception of virtual networks), however it has been confirmed through numerous evaluations that the analytical model holds for other non-homogeneous system parameters. 


\section{RESUlts AND SyStem CHARACTERISTICS}

\section{A. Classic Network Baseline}

The case of a single classic network was used to develop a baseline to which CR improvements could be compared. Results from the single network case can easily be extended to multiple networks as the networks are, by definition, independent of each other.

It is assumed that network service provider must deliver a certain level of QoS, based on the $P_{F}$ (which for the classical networks is equal to $P_{B}$ ). It is then important to know the traffic intensity $(\rho=\lambda / \mu)$ that can be supported by the network while achieving this QoS. Fig. 2 shows the maximum traffic intensities supported while limiting blocking to either $1 \%$ or $5 \%$. Results for networks with between 1 and 100 channels are shown.

\section{B. Two Network Scenario}

With the basic classic network case established, networks with cognitive abilities can now be explored. This exploration begins with the simplest cases involving only two networks. We will study the case where both networks utilize cognitive radios.

If the networks carry the baseline traffic intensity, the resulting performance is very similar to the cognitive network in the previous 2-network scenario. As even the classic failure rate was very low (1\%), the change to cognitive radio users has very little effect on the overall utilization.

As in the previous scenario, if traffic is increased subject to the $1 \%$ constraints on $P_{F}$, then the maximum supportable traffic can be found for a certain QoS level. In this scenario, traffic can now be adjusted on both CR networks. As shown in Fig. 3 , there is a significant improvement over the classic baseline performance, although the relative gains are most pronounced in small networks.

Clearly, the cognitive systems are a major improvement over the classic networks. The primary drawback is the resulting dropping of flows. This is a direct consequence of the cognitive radio requirement that cognitive users cannot interfere with primary users, so if a primary flow arrives, it has priority over a cognitive flow. However, despite the drops, the overall $P_{F}$ is far better when $\mathrm{CR}$ is enabled.

\section{Virtual Networks}

To take advantage of some of this remaining unutilized bandwidth, virtual networks are now introduced to the scenario.

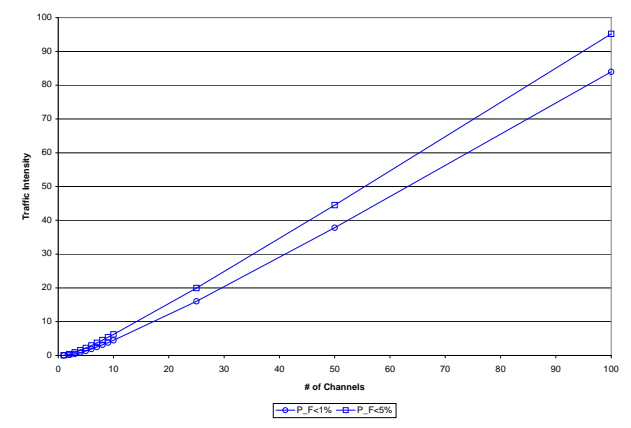

Fig. 2. Maximum Traffic Intensities at $P_{F} \leq 1 \%, 5 \%$ - Single Network

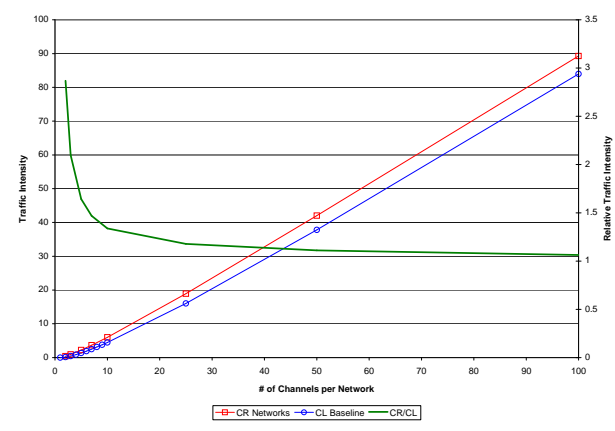

Fig. 3. Relative Traffic Intensity - 2 CR vs. Baseline CL

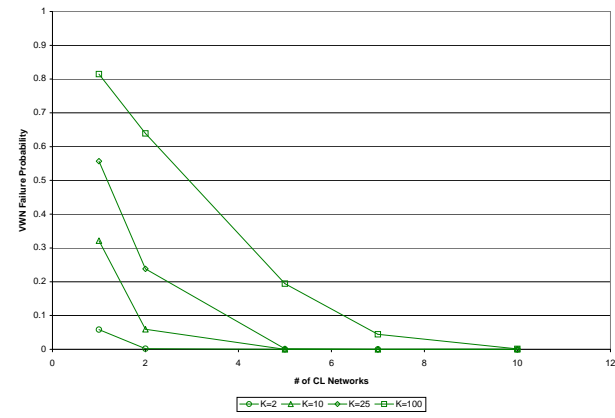

Fig. 4. QoS Levels Provided by VWN- Varying Number of CL Networks

The simplest scenario involving a virtual network is the system consisting of one classic network and a virtual network. If the classic network traffic is already at the maximum traffic intensity for a particular QoS level (e.g., traffic intensity for $\leq 1 \%$ blocking), then obviously the virtual network cannot make the same QoS guarantee. One possibility is to simply support additional traffic, without any QoS guarantees - this is often the approach suggested in CR.

Consider the scenario where there are $n$ independent classic networks, each supporting the maximum classic traffic for $P_{F} \leq 1 \%$ blocking. While any additional classic traffic would increase the $P_{F}$ above the $1 \%$ constraint, if an additional virtual network is added using CR-techniques, it can make use of unused capacity on any of the existing networks, without affecting the $P_{F}$ constraint of the classic networks. Fig. 4 shows the resulting QoS for a VWN supporting the same baseline intensity as the classic networks.

As can be seen in the figure, the resulting performance depends on both the number of networks and the number of channels per network. Recall that the baseline traffic intensity increases as the number of channels $K$ increases. Therefore, Fig. 4 shows two important properties. First, as the number of classic networks increases, $P_{F}(V W N)$ decreases, as there is an increased probability that one of the networks has available resources. Second, increases in network size (number of channels) result in an increase in the $P_{F}(V W N)$. This is due to the fact that larger classic networks (greater $K$ ) have a higher baseline utilization level than smaller networks, leaving a lower probability of having resources available for cognitive use.

Fig. 5 shows the maximum traffic intensity that can be supported on the virtual network while maintaining a QoS level 


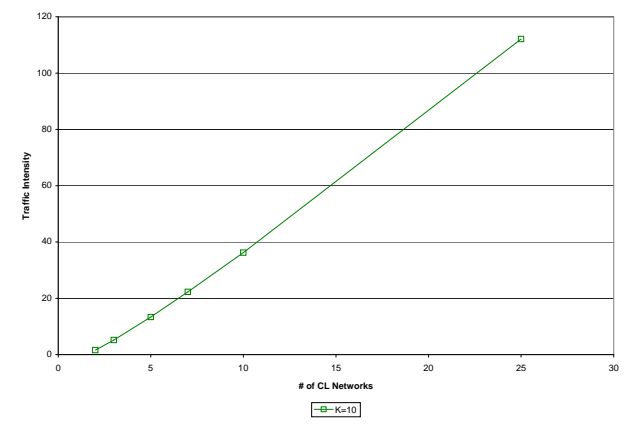

Fig. 5. Max VWN Traffic Intensity for $1 \%$ Failures

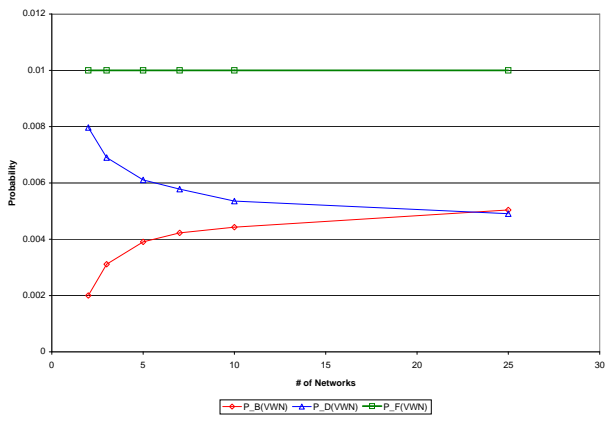

Fig. 6. Behaviour of the VWN at Max Traffic Intensity, $P_{F} \leq 1 \%$

(the usual $1 \%$ failure rate). Again, all classic networks continue to maintain the baseline traffic intensity and QoS performance. In this case, each classic network possesses $K=10$ channels. Considering that the baseline intensity for $K=10$ is $\rho=4.48$ (from Fig. 2), for the 25-network system, the VWN actually carries a traffic intensity of $\rho=112$. This is the equivalent traffic of 25 additional classic networks. Although this is not quite as efficient as the equivalent single network (all channels and all traffic accessing one network - i.e. $N=1, K=250$ ), it is a tremendous improvement over multiple classical networks.

The QoS behaviour of these networks is interesting. If the $P_{B}, P_{D}$, and $P_{F}$ are plotted for this virtual network, a shift can be seen. Fig. 6 shows that if the VWN is carrying the maximum allowable traffic, the cause of failures shifts from mostly dropping to an even split of blocking and dropping, as the number of networks increases. In networks where drops are considered more problematic (or costly) than blocks, this may be an important characteristic to consider.

\section{CONCLUSION}

This work has focused on studying the benefits to QoS reliability that can be gained by adding cognitive radio capabilities to a system of classical, connection-oriented wireless networks. The resulting cognitive radio system has been modelled, analyzed, simulated, studied.

The system model developed describes a collection of independent service providers, and its clients. The system is by a Markov process, with continuous time and finite state space. Using this Markov process, the system was analyzed to find expressions for the classical QoS reliability metrics - connection blocking and dropping. Simulations were also prepared and shown to closely agree with the analytical model. Using these two tools, a number of experiments were performed, in order to evaluate the characteristics of such a system.

Through these experiments, the potential performance gains of applying cognitive radio has been quantified. It has been verified that cognitive radio can allow for an improvement in QoS or the support of additional best-effort users without deteriorating the QoS of existing users.

It has also been shown that through the creation of virtual wireless networks, additional clients can be supported with QoS guarantees. This is made possible by the VWN's ability to make use of resources from all of the existing networks. The experiments performed have also quantified the maximum supportable traffic intensity that can be supported while maintaining a particular failure probability.

\section{REFERENCES}

[1] M. A. McHenry, et. al., Chicago spectrum occupancy measurements \& analysis and a long-term studies proposal. In proc. of ACM $1^{\text {st }}$ International workshop on Technology and policy for accessing spectrum, 2006.

[2] FCC Spectrum Policy Task Force Report. Federal Communication Commission (USA), ET Docket No. 02-135, Nov. 2002.

[3] J. Mitola III, Cognitive Radio: An Integrated Agent Architecture for Software Defined Radio. PhD Thesis, KTH Royal Institute of Technology, Stockholm, Sweden, 2000.

[4] S. Haykin, Cognitive radio: brain-empowered wireless communications. IEEE Journal on Selected Areas in Communications, vol. 23, no. 2, pp. 201-220, Feb. 2005.

[5] C. Cordeiro, et. al., IEEE 802.22: the first worldwide wireless standard based on cognitive radios. In proc. of $1^{\text {st }}$ IEEE International Symposium on New Frontiers in Dynamic Spectrum Access Networks, pp. 328-337, Nov. 2005.

[6] M. Much, et. al., IEEE P1900.B: Coexistence Support for Reconfigurable, Heterogeneous Air Interfaces. In proc. of $2^{\text {nd }}$ IEEE International Symposium on New Frontiers in Dynamic Spectrum Access Networks, pp. 381-389, Apr. 2007.

[7] N. Jesuale, Spectrum policy issues for state and local government. International Journal of Network Management (Wiley), vol. 16, no. 2, pp. 89-101, Mar. 2006.

[8] J. A. Hoffmeyer, Regulatory and standardization aspects of DSA technologies - global requirements and perspective. In proc. of $2^{\text {nd }}$ IEEE International Symposium on New Frontiers in Dynamic Spectrum Access Networks, pp. 700-705, Apr. 2007.

[9] N. Devroye, P. Mitran, and V. Tarokh, Achievable Rates in Cognitive Radio Channels. IEEE Transactions on Information Theory, vol. 52, no. 5, pp.1813-1827, May 2006.

[10] S. A. Jafar and S. Srinivasa, Capacity Limits of Cognitive Radio With Distributed and Dynamic Spectral Activity. IEEE Journal on Selected Areas in Communications, vol. 25, no. 3, pp. 529-537, Apr. 2007.

[11] T. Weiss and F. K. Jondral, Spectrum pooling: an innovative strategy for the enhancement of spectrum efficiency. IEEE Communications Magazine, vol. 42, no. 3, pp. S8-14, Mar. 2004.

[12] F. Capar, et. al., Comparison of bandwidth utilization for controlled and uncontrolled channel assignment in a spectrum pooling system. In proc. of IEEE $55^{\text {th }}$ Vehicular Technology Conference, vol. 3, pp. 1069-1073, 2002.

[13] B. Wang, Z. Ji, and K. J. R. Liu, Primary-Prioritized Markov Approach for Dynamic Spectrum Access. In proc. of $2^{\text {nd }}$ IEEE International Symposium on New Frontiers in Dynamic Spectrum Access Networks, pp. 507-515, Apr. 2007.

[14] D. Cabric, et. al., A Cognitive Radio Approach for Usage of Virtual Unlicensed Spectrum. In proc. of $14^{\text {th }}$ IST Mobile Wireless Communications Summit, Dresden, Germany, June 2005.

[15] C. E. Caicedo and M. B. H. Weiss, Spectrum Trading: An Analysis of Implementation Issues. In proc. of $2^{\text {nd }}$ IEEE International Symposium on New Frontiers in Dynamic Spectrum Access Networks, pp. 579-584, Apr. 2007. 